International Journal of Pure and Applied Mathematics

Volume 83 No. 2 2013, 221-231

ISSN: $1311-8080$ (printed version); ISSN: 1314-3395 (on-line version)

url: http://www.ijpam.eu

doi: http://dx.doi.org/10.12732/ijpam.v83i2.1

ijpam.eu

\title{
PRIME GAMMA NEAR-RINGS WITH DERIVATIONS
}

\author{
Isamiddin S. Rakhimov ${ }^{1}$, Kalyan Kumar Dey ${ }^{2}$, Akhil Chandra Paul ${ }^{3}$ \\ ${ }^{1}$ Department of Mathematics \\ FS, \& Institute for Mathematical Research (INSPEM) \\ Universiti Putra Malaysia \\ MALAYSIA \\ ${ }^{2,3}$ Department of Mathematics \\ Rajshahi University \\ Rajshahi, 6205, BANGLADESH
}

Abstract: Let $N$ be a prime $\Gamma$ near-ring with the center $Z(N)$. The objective of this paper is to study derivations on $N$. We prove two results:

(a) Let $N$ be 2-torsion free and let $D_{1}$ and $D_{2}$ be derivations on $N$ such that $D_{1} D_{2}$ is also a derivation. Then $D_{1}=0$ or $D_{2}=0$ if and only if $\left[D_{1}(x), D_{2}(y)\right]_{\alpha}=0$ for all $x, y \in N, \alpha \in \Gamma$;

(b) Let $n$ be an integer greater than $1, N$ be $n$ !-torsion free, and $D$ be a derivation with $D^{n}(N)=\{0\}$. Then $D(Z(N))=\{0\}$.

AMS Subject Classification: 16W25, 16Y30, 16Y99

Key Words: commutative ring, non commutative ring, derivation

\section{Introduction}

The notion of derivations in near-rings has been introduced by Bell and Mason [2]. They obtained some basic properties of derivations in near-rings. Then Asci [1] investigated commutativity conditions for a $\Gamma$-near-ring with derivations. Cho and Jun [7] studied some characterizations of $\Gamma$-near-rings and some regularity conditions. In classical ring theory, Posner [14], Herstein [11], Bergen

Received: June 13, 2012

(c) 2013 Academic Publications, Ltd.

$\S$ Correspondence author url: www.acadpubl.eu 
[5], Bell and Daif [4] studied derivations in prime and semiprime rings and obtained commutativity results of prime and semiprime rings with derivations. In near ring theory, Bell and Mason [3], and also Cho [8] worked on derivations in prime and semiprime near-rings.

In this paper, we deal with the prime $\Gamma$-near-rings with derivations. Here we extend the results of Wang [20] on prime near-rings of to $\Gamma$-near-rings.

\section{Preliminaries}

A $\Gamma$-near-ring is a triple $(N,+, \Gamma)$, where:

(i) $(N,+)$ is a group (not necessarily abelian),

(ii) $\Gamma$ is a non-empty set of binary operations on $N$ such that for each $\alpha \in \Gamma$,

$(N,+, \alpha)$ is a left near-ring.

(iii) $x \alpha(y \beta z)=(x \alpha y) \beta z$,for all $x, y, z \in N$ and $\alpha, \beta \in \Gamma$.

Examples of $\Gamma$-near-rings and motivations to study has been given in $[16$, $17]$.

Throughout this paper, $N$ will denote a zero-symmetric left $\Gamma$-near-ring with multiplication center $\mathrm{Z}(\mathrm{N})$. A $\Gamma$-near-ring $N$ is called a prime $\Gamma$-near-ring if $N$ has the property that for $\mathrm{x}, \mathrm{y} \in N, x \Gamma N \Gamma y=\{0\}$ implies $x=0$ or $y=$ 0 . A $\Gamma$-near-ring $N$ is called a semiprime $\Gamma$-near-ring if $N$ has the property that for $x \in N, x \Gamma N \Gamma x=\{0\}$ implies $x=0$. A derivation $D$ on $N$ is an additive endomorphism of $N$ with the property that for all $x, y \in N$ and $\alpha \in \Gamma, D(x \alpha y)=x \alpha D(y)+D(x) \alpha y$. An additive endomorphism $D$ of $N$ is called a derivation on $N$ if $D(x \alpha y)=x \alpha D(y)+D(x) \alpha y$ for all $x, y \in N, \alpha \in \Gamma$ A $\Gamma$-near-ring $N$ is called commutative if $(N,+)$ is abelian, and 2-torsion free if $2 x=0$ implies $x=0$.

\section{Derivations in Prime $\Gamma$-Near-Rings}

We begin with the following lemmas on derivations on prime $\Gamma$-near-rings $N$.

Lemma 3.1. Let $D$ be an additive endomorphism of $N$. Then:

$$
D(x \alpha y)=x \alpha D(y)+D(x) \alpha y
$$

for all $x, y \in N$ and $\alpha \in \Gamma$, if and only if

$$
D(x \alpha y)=D(x) \alpha y+x \alpha D(y)
$$


for all $x, y \in N$ and $\alpha \in \Gamma$.

Proof. We assume that $D(x \alpha y)=x \alpha D(y)+D(x) \alpha y$ for all $x, y \in N$ and $\alpha \in \Gamma$.

Since

$$
x \alpha(y+y)=x \alpha y+x \alpha y
$$

and

$D(x \alpha(y+y))=x \alpha D(y+y)+D(x) \alpha(y+y)=x \alpha D(y)+x \alpha D(y)+D(x) \alpha y+D(x) \alpha y$ and

$D(x \alpha y+x \alpha y)=D(x \alpha y)+D(x \alpha y)=x \alpha D(y)+D(x) \alpha y+x \alpha D(y)+D(x) \alpha y$

we get

$$
x \alpha D(y)+D(x) \alpha y=D(x) \alpha y+x \alpha D(y),
$$

so

$$
D(x \alpha y)=D(x) \alpha y+x \alpha D(y),
$$

for all $x, y \in N \alpha \in \Gamma$.

The converse is proved in a similar way.

Note that due to Lemma 3.1, $D$ is a derivation if and only if

$$
D(x \alpha y)=D(x) \alpha y+x \alpha D(y) \text {, for all } x, y \in N \text { and } \alpha \in \Gamma .
$$

We make use the following lemma from [15], Lemma 3.5.

Lemma 3.2. Suppose that $N$ is a prime $\Gamma$-near-ring.

(i) any nonzero element of the center of $N$ is not zero divisor.

(ii) If there exist a nonzero element of $Z(N)$ such that $x+x \in Z(N)$, then $(N,+)$ is commutative.

(iii) Let dbe a nonzero derivation on $N$. If one of the $x \Gamma d(N)=\{0\}$ and $d(N) \Gamma x=\{0\}$ holds then $x=0$.

Lemma 3.3. Let $D$ be a derivation on $N$. Then $N$ satisfies the following partial distributive laws

(i) $(x \alpha D(y)+D(x) \alpha y) \beta z=x \alpha D(y) \beta z+D(x) \alpha y \beta z$ for all $x, y, z \in N$ and $\alpha, \beta \in \Gamma$

(ii) $(D(x) \alpha y+x \alpha D(y)) \beta z=D(x) \alpha y \beta z+x \alpha D(y) \beta z$ for all $x, y, z \in N$ and $\alpha, \beta \in \Gamma$ 
Proof. (i) Consider $D((x \alpha y) \beta z)=D(x \alpha(y \beta z))$ for all $x, y, z \in N$ and $\alpha$, $\beta \in \Gamma$. Then by using Lemma 3.1, we obtain the required result.

(ii) Consider $D((x \alpha y) \beta z)=D(x \alpha(y \beta z)$. Then we make use Lemma 3.1 to obtain,

$$
D((x \alpha y) \beta z)=D(x \alpha y) \beta z+x \alpha y \beta D(z)=(D(x) \alpha y+x \alpha D(y)) \beta z+x \alpha y \beta D(z)
$$

and

$$
\begin{aligned}
& D(x \alpha(y \beta z))=D(x) \alpha y \beta z+x \alpha D(y \beta z) \\
& =D(x) \alpha y \beta z+x \alpha(D(y) \beta z+y \beta D(z))=D(x) \alpha y \beta z+x \alpha D(y) \beta z+x \alpha y \beta D(z),
\end{aligned}
$$

for all $x, y, z \in N$ and $\alpha, \beta \in \Gamma$.

Comparing the above two relations we get the required result:

$$
(D(x) \alpha y+x \alpha D(y)) \beta z=D(x) \alpha y \beta z+x \alpha D(y) \beta z
$$

for all $x, y, z \in N$ and $\alpha, \beta \in \Gamma$.

Now we prove our main results.

Theorem 3.4. Let $N$ be a 2-torsion-free prime $\Gamma$ near-ring, and let $D_{1}$ and $D_{2}$ be derivations on $N$ such that $D_{1} D_{2}$ is also a derivation. Then the following two conditions are equivalent:

1. either $D_{1}=0$ or $D_{2}=0$;

2. (ii) $\left[D_{1}(x), D_{2}(y)\right]_{\alpha}=0$ for all $x y \in N, \alpha \in \Gamma$

Proof. We need prove only the part (ii) $\Rightarrow$ (i) since (i) $\Rightarrow$ (ii) is obvious. Consider

$$
D_{1} D_{2}(x \alpha y)=x \alpha D_{1} D_{2}(y)+D_{1} D_{2}(x) \alpha y
$$

for all $x, y, z \in N$ and $\alpha \in \Gamma$.

On the other hand, both $D_{1}$ and $D_{2}$ are derivations, therefore

$$
\begin{aligned}
D_{1} D_{2}(x \alpha y) & =D_{1}\left(D_{2}(x \alpha y)\right) \\
& =D_{1}\left(x \alpha D_{2}(y)+D_{2}(x) \alpha y\right) \\
& =D_{1}\left(x \alpha D_{2}(y)\right)+D_{1}\left(D_{2}(x) \alpha y\right) \\
& =x \alpha D_{1} D_{2}(y)+D_{1}(x) \alpha D_{2}(y)+D_{2}(x) \alpha D_{1}(y)+D_{1} D_{2}(x) \alpha y,
\end{aligned}
$$

for all $x, y \in N$ and $\alpha \in \Gamma$. 
The above two relations for $D_{1} D_{2}(x \alpha y)$ give

$$
D_{1}(x) \alpha D_{2}(y)+D_{2}(x) \alpha D_{1}(y)=0 \text { for all } x, y \in N \text { and } \alpha \in \Gamma \text {. }
$$

Replacing $x$ by $x \beta D_{2}(z), z \in N, \beta \in \Gamma$ in (1), by using Lemma 31 and Lemma 3.3 we get

$$
\begin{aligned}
0= & D_{1}\left(x \beta D_{2}(z)\right) \alpha D_{2}(y)+D_{2}\left(x \beta D_{2}(z)\right) \alpha D_{1}(y) \\
= & \left(D_{1}(x) \beta D_{2}(z)+x \beta D_{1} D_{2}(z)\right) \alpha D_{2}(y)+\left(x \beta D_{2}^{2}(z)+D_{2}(x) \beta D_{2}(z)\right) \alpha D_{1}(y) \\
= & D_{1}(x) \beta D_{2}(z) \alpha D_{2}(y)+x \beta D_{1} D_{2}(z) \alpha D_{2}(y)+x \beta D_{2}^{2}(z) \alpha D_{1}(y) \\
& +D_{2}(x) \beta D_{2}(z) \alpha D_{1}(y) \\
= & D_{1}(x) \beta D_{2}(z) \alpha D_{2}(y)+x \beta\left(D_{1} D_{2}(z) \alpha D_{2}(y)+D_{2}^{2}(z) \alpha D_{1}(y)\right) \\
& +D_{2}(x) \beta D_{2}(z) \alpha D_{1}(y),
\end{aligned}
$$

for all $x, y, z \in N$ and $\alpha, \beta \in \Gamma$.

Then by using the equation (1) we obtain

$$
x \beta\left(D_{1} D_{2}(z) \alpha D_{2}(y)+D_{2}^{2}(z) \alpha D_{1}(y)\right)=0 .
$$

If we replace $x$ by $D_{2}(z)$ in (1) then we get

$$
D_{1} D_{2}(z) \alpha D_{2}(y)+D_{2}^{2}(z) \alpha D_{1}(y)=0 .
$$

Therefore

$$
\begin{aligned}
& D_{1}(x) \beta D_{2}(z) \alpha D_{2}(y)+D_{2}(x) \beta D_{2}(z) \alpha D_{1}(y)=0 \\
& \qquad \text { for all } x, y, z \in N \text { and } \alpha, \beta \in \Gamma .
\end{aligned}
$$

Replacing $x$ and $y$ by $z$ in (1), respectively, we obtain

$$
D_{2}(z) \alpha D_{1}(y)=-D_{1}(z) \alpha D_{2}(y) \text { for all } y, z \in N \text { and } \alpha \in \Gamma,
$$

and

$$
D_{1}(x) \alpha D_{2}(z)=-D_{2}(x) \alpha D_{1}(z) \text { for all } x, z \in N \text { and } \alpha \in \Gamma .
$$

Since $N$ is a zero-symmetric left $\Gamma$ near-ring, then due to (2) we obtain

$$
\begin{aligned}
0 & =\left(-D_{2}(x) \beta D_{1}(z)\right) \alpha D_{2}(y)+D_{2}(x) \beta\left(-D_{1}(z) \alpha D_{2}(y)\right) \\
& =D_{2}(x) \beta\left(-D_{1}(z)\right) \alpha D_{2}(y)+D_{2}(x) \beta\left(-D_{1}(z) \alpha D_{2}(y)\right) \\
& =D_{2}(x) \beta\left[\left(-D_{1}(z)\right) \alpha D_{2}(y)-D_{1}(z) \alpha D_{2}(y)\right]
\end{aligned}
$$


for all $x, y, z \in N$ and $\alpha, \beta \in \Gamma$.

If $D_{2} \neq 0$ then thanks to Lemma 3.2 we have

$$
\left(-D_{1}(z)\right) \alpha D_{2}(y)-D_{1}(z) \alpha D_{2}(y)=0 .
$$

That is

$$
D_{1}(z) \alpha D_{2}(y)=\left(-D_{1}(z)\right) \alpha D_{2}(y) \text { for all } y, z \in N \text { and } \alpha \in \Gamma .
$$

The condition (ii) provides

$$
\begin{aligned}
& \left(-D_{1}(z)\right) \alpha D_{2}(y)=D_{1}(-z) \alpha D_{2}(y)=D_{2}(y) \alpha D_{1}(-z) \\
= & D_{2}(y) \alpha\left(-D_{1}(z)\right)=-D_{2}(y) \alpha D_{1}(z)=-D_{1}(z) \alpha D_{2}(y) .
\end{aligned}
$$

Therefore

$$
\left(-D_{1}(z)\right) \alpha D_{2}(y)=-D_{1}(z) \alpha D_{2}(y) \text { for all } y, z \in N \text { and } \alpha \in \Gamma \text {. }
$$

From (3) and (4) we obtain $2 D_{1}(z) \alpha D_{2}(y)=0$ for all $y, z \in N$ and $\alpha \in \Gamma$ Since $N$ is 2-torsion-free, this gives $D_{1}(z) \alpha D_{2}(y)=0$ for all $y, z \in N$ and $\alpha \in \Gamma$ Therefore $D_{1}(z) \alpha D_{2}(N)=\{0\}$. But $D_{2} \neq 0$, so $D_{1}(z)=0$ for all $z \in N$, that is $D_{1}=0$.

Note that Lemma 3.5. from [15] can be derived now as the following corollary from the theorem.

Corollary 3.5 Let $N$ be a 2-torsion free prime Tnear-ring, and let $D$ be a derivation on $N$ such that $D^{2}=0$. Then $D=0$.

Proof. It is clear that $D^{2}=0$ is a derivation on $N$, and we have

$$
\begin{aligned}
& 0=D^{2}(x \alpha y)=D(x \alpha D(y)+D(x) \alpha y)=D(x \alpha D(y))+D(D(x) \alpha y) \\
& \quad=x \alpha D^{2}(y)+D(x) \alpha D(y)+D(x) \alpha D(y)+D^{2}(x) \alpha y=2 D(x) \alpha D(y)
\end{aligned}
$$

for all $x, y \in N$ and $\alpha \in \Gamma$. Since $N$ is 2-torsion free we obtain $D(x) \alpha D(y)=$ 0 for all $x, y \in N$ and $\alpha \in \Gamma$ Similarly we get $D(y) \alpha D(x)=0$ for all $x, y \in N$ and $\alpha \in \Gamma$ Therefore $[D(x), D(y)]_{\alpha}=0$ for all $x, y \in N$ and $\alpha \in \Gamma$. Hence by Theorem 3.4, we get $D=0$.

Another consequence of Theorem 3.4 is the following

Corollary 3.6. Let $N$ be a Tnear-ring and $D_{1}$ and $D_{2}$ be derivations on $N$ such that $D_{1} D_{2}$ is a derivation. Then $D_{2} D_{1}$ is also a derivation. 
Proof. Obviously $D_{2} D_{1}$ is an additive endomorphism of $N$. By Theorem 3.4 we have

$$
\begin{aligned}
D_{2} D_{1}(x \alpha y) & =D_{2}\left(D_{1}(x) \alpha y+x \alpha D_{1}(y)\right)=D_{2}\left(D_{1}(x) \alpha y\right)+D_{2}\left(x \alpha D_{1}(y)\right) \\
& =D_{2} D_{1}(x) \alpha y+\left(D_{1}(x) \alpha D_{2}(y)+D_{2}(x) \alpha D_{1}(y)\right)+x \alpha D_{2} D_{1}(y) \\
& =D_{2} D_{1}(x) \alpha y+x \alpha D_{2} D_{1}(y)
\end{aligned}
$$

for all $x y \in N$ and $\alpha \in \Gamma$.

This completes the proof.

The following is an extension of Wang [20] on Leibniz's rule for derivations of rings to Tnear-rings.

Theorem 3.7. Let $N$ be a $n$ !-torsion free $\Gamma$-near-ring Let $n$ be an integer $n \geq 2$ and $D$ be a derivation on $N$. Then

$$
\begin{array}{r}
D^{n}(x \alpha y)=D^{n}(x) \alpha y+\left(\begin{array}{c}
n \\
1
\end{array}\right) D^{n-1}(x) \alpha D(y)+\cdots+\left(\begin{array}{c}
n \\
i
\end{array}\right) D^{n i}(x) \alpha D^{i}(y) \\
+\cdots+\left(\begin{array}{l}
n \\
n-1
\end{array}\right) D(x) \alpha D^{n-1}(y)+x \alpha D^{n}(y)
\end{array}
$$

for all $x, y \in N$ and $\alpha \in \Gamma$.

Proof. By Theorem 3.4 it can easily seen that

$$
D(x) \alpha y+n x \alpha D(y)=n x \alpha D(y)+D(x) \alpha y
$$

for all $x, y \in N, \alpha \in \Gamma$ and $n$ be an integer. The same observation gives

$$
\begin{aligned}
n D(x) \alpha y+n x \alpha D(y)= & n(D(x) \alpha y+x \alpha D(y)) \\
& \text { for all } x, y \in N, \alpha \in \Gamma, \text { and } n \text { be an integer. }
\end{aligned}
$$

We proceed the proof of Leibniz's rule by induction on $n$. Let $n=2$. Then

$$
\begin{aligned}
D^{2}(x \alpha y) & =D(D(x) \alpha y+x \alpha D(y)) \\
& =D(D(x) \alpha y)+D(x \alpha D(y)) \\
& =D^{2}(x) \alpha y+D(x) \alpha D(y)+D(x) \alpha D(y)+x \alpha D^{2}(y) \\
& =D^{2}(x) \alpha y+2 D(x) \alpha D(y)+x \alpha D^{2}(y) .
\end{aligned}
$$

Assume that Leibniz's rule holds for $n-1$. That is, if $N$ is $(n-l)$ !-torsion-free.

Then 


$$
\begin{aligned}
D^{n-1}(x \alpha y)=D^{n-1}(x) \alpha y & +\cdots+\left(\begin{array}{c}
n-1 \\
i-1
\end{array}\right) D^{n i}(x) \alpha D^{i-1}(y) \\
& +\left(\begin{array}{c}
n-1 \\
i
\end{array}\right) D^{n i-1}(x) \alpha D^{i}(y)+\cdots+x \alpha D^{n-1}(y) .
\end{aligned}
$$

Since $n$ !-torsion-freeness implies $(n-l)$ !-torsion-freeness, by $(5)$ we have

$$
\begin{aligned}
& D^{n}(x \alpha y)=D\left(D^{n-1}(x \alpha y)\right) \\
& =D\left(D^{n-1}(x) \alpha y+\ldots+\left(\begin{array}{c}
n-1 \\
i-1
\end{array}\right) D^{n i}(x) \alpha D^{i-1}(y)+\left(\begin{array}{l}
n-1 \\
i
\end{array}\right) D^{n i-1}(x) \alpha D^{i}(y)\right. \\
& \left.+\cdots+x \alpha D^{n-1}(y)\right) \\
& =D\left(D^{n-1}(x) \alpha y+\ldots+\left(\begin{array}{c}
n-1 \\
i-1
\end{array}\right) D\left(D^{n i}(x) \alpha D^{i-1}(y)\right)\right. \\
& +\left(\begin{array}{l}
n-1 \\
i
\end{array}\right) D\left(D^{n i-1}(x) \alpha D^{i}(y)\right)+\cdots+D\left(x \alpha D^{n-1}(y)\right) \\
& =D^{n}(x) \alpha y+D^{n-1}(x) \alpha D(y)+\cdots+\left(\left(\begin{array}{c}
n-1 \\
i-1
\end{array}\right) D^{n i+1}(x) \alpha D^{i-1}(y)+D^{n i}(x) \alpha D^{i}(y)\right) \\
& +\left(\begin{array}{l}
n-1 \\
i
\end{array}\right)\left(D^{n i}(x) \alpha D^{\mathrm{i}}(y)+D^{n i-1}(x) \alpha D^{i+1}(y)\right)+\cdots++D(x) \alpha D^{n-1}(y)+x \alpha D^{n}(y) \\
& =D^{n}(x) \alpha y+\left(\begin{array}{c}
n-1 \\
i-1
\end{array}\right) D^{n i+1}(x) \alpha D^{i-1}(y)+\left(\begin{array}{c}
n-1 \\
i-1
\end{array}\right) D^{n i}(x) \alpha D^{i}(y) \\
& +\left(\begin{array}{l}
n-1 \\
i
\end{array}\right) D^{n i}(x) \alpha D^{i}(y)+\left(\begin{array}{l}
n-1 \\
i
\end{array}\right)\left(D^{n i-1}(x) \alpha D^{i+1}(y)+\cdots+x \alpha D^{n}(y)\right. \\
& =D^{n}(x) \alpha y+\cdots+\left(\begin{array}{c}
n-1 \\
i-1
\end{array}\right) D^{n i}(x) \alpha D^{i}(y)+\left(\begin{array}{l}
n-1 \\
i
\end{array}\right) D^{n i}(x) \alpha D^{i}(y) \\
& +\cdots+x \alpha D^{n}(y) \\
& =D^{n}(x) \alpha y+\cdots+\left[\left(\begin{array}{l}
n-1 \\
i-1
\end{array}\right)+\left(\begin{array}{l}
n-1 \\
i
\end{array}\right)\right] D^{n i}(x) \alpha D^{i}(y)+\cdots \cdots+x \alpha D^{n}(y) \\
& =D^{n}(x) \alpha y+\cdots+\left(\begin{array}{c}
n \\
i
\end{array}\right) D^{n i}(x) \alpha D^{i}(y)+\cdots \cdots+x \alpha D^{n}(y)
\end{aligned}
$$

The proof is complete.

Lemma 3.8. Let $N$ be a Tnear-ring with center $Z(N)$, and let $D$ be a derivation on $N$. Then $D(Z(N)) \subseteq Z(N)$.

Proof. By Theorem 3.4, we have 


$$
\begin{aligned}
x \alpha D(z)+z \alpha D(x)=x \alpha D(z)+D(x) \alpha z=D(x \alpha z)= & D(z \alpha x) \\
& =D(z) \alpha x+z \alpha D(x)
\end{aligned}
$$

for all $z \in Z(N), x \in N$ and $\alpha \in \Gamma$.

Therefore $x \alpha D(z)=D(z) \alpha x$ for all $x, z \in N$ and $\alpha \in \Gamma$. Thus $D(z) \in Z(N)$

Lemma 3.9. Let $n \geq 2$, and let $N$ be an $n$ !-torsion free $\Gamma$ near-ring and $D$ be a derivation with $D^{n}(N)=\{0\}$. Then for each $y \in N$, either $D(y)=0$ or there exists $k(0<k<n)$ such that $D^{k}(y)$ is a nonzero divisor of zero.

Proof. Since $n$ !-torsion-freeness implies $(n-1)$ !-torsion-freeness, we may assume that $D^{n-1}(N) \neq\{0\}$ Choose $x$ such that $D^{n-1}(x) \neq 0$. Assume that $D(y) \neq 0$. Then there exists $k$ with $0<k<n$ such that $D^{k}(y) \neq 0$ and $D^{k+1}(y)=0$. Then due to Theorem 3.7 we obtain

$$
\begin{gathered}
0=D^{n}\left(x \alpha D^{k 1}(y)\right)=D^{n}(x) \alpha D^{k-1}(y)+\left(\begin{array}{c}
n \\
1
\end{array}\right) D^{n-1}(x) \alpha D^{k}(y) \\
+\left(\begin{array}{c}
n \\
2
\end{array}\right) D^{n-2}(x) \alpha D^{k+1}(y)+\cdots=\left(\begin{array}{c}
n \\
1
\end{array}\right) D^{n-1}(x) \alpha D^{k}(y)=n D^{n-1}(x) \alpha D^{k}(y)
\end{gathered}
$$

for all $y \in N$ and $\alpha \in \Gamma$.

Since $N$ is $n$ !-torsion-free, then we get $D^{n-1}(x) D^{k}(y)=0$ for all $y \in N$ and $\alpha \in \Gamma$. By Lemma 3.2(i) $D^{k}(y)$ is a nonzero divisor of zero.

We finalize the paper by the following two theorems which can be easily proven by using the previous results

Theorem 3.10. Let $n$ be an integer $\geq 1$ and $N$ be a prime Tnear-ring with center $Z(N)$, and let $N$ be $n$ !-torsion free and $D$ be a derivation with $D^{n}(N)=\{0\}$. Then $D(Z(N))=\{0\}$.

Theorem 3.11. Let $n$ be a positive integer and $N$ be an $n$ !-torsion free Tnear-ring with no divisor of zero, then $N$ admits no nonzero derivation $D$ with $D^{n}=0$.

\section{Acknowledgments}

The second named author thanks the Institute for Mathematical Research (INSPEM), UPM, Malaysia for the hospitality during that this paper has been written.

The research was supported by FRGS grant 01-12-10978FR MOHE, Malaysia. 


\section{References}

[1] M. Asci, $\Gamma-(\sigma, \tau)$-derivation on gamma near ring, Int. Math. Forum, 2, No. 3 (2007), 97-102.

[2] H.E. Bell, G. Mason, On derivations in near-ring, near-rings and nearfields, North-Holland, Math. Studies, 137 (1987), 31-35.

[3] H.E. Bell, G. Mason, On derivations in near-rings and rings, Math. J. Okayama Univ., 34 (1992), 135-144.

[4] H.E. Bell, M.N. Daif, On derivations and commutativity in prime rings, Acta. Math. Hungar., 66, No. 4 (1995), 337-343.

[5] J. Bergen, Derivations in prime rings, Canad. Math. Bull., 26, No. 3 (1983), 267-227.

[6] G.L. Booth, A note on $\Gamma$-near rings, Studia Sci. Math. Hungarica, 23 (1988), 471-475

[7] Y.U. Cho, Y.B. Jun, Gamma-near-rings with gamma derivations, Pure and Appl. Indian Math., 33, No. 10 (2002), 1489-1494.

[8] Y.U. Cho, Some conditions on derivations in prime near rings, J. Korea Soc. Math. Educ. Ser. B Pure Appl. Math., 8, No. 2 (2001), 145-152.

[9] Y.U. Cho, A study on derivations in near-rings, Pusan Kyongnam Math. J., 12, No. 1 (1996), 63-69.

[10] K.K. Dey, A.C. Paul, I.S. Rakhimov, On prime gamma-near-rings with generalized derivations, International Journal of Math. and Math. Sci., 2012, doi: 10.1155/2012/625968.

[11] I.N. Herstein, A note on derivations, Canad. Math. Bull., 21, No. 3 (1978), 369-370.

[12] Y. B. Jun, K. H. Kim and Y. U. Cho, On gamma-derivation in gammanear-rings, Soochow J. Math., 29, No. 3 (2003), 275-282.

[13] G. Pilz, Near-rings, North-Holland Mathematics Studies, 23 (1983).

[14] E.C. Posner, Derivations in prime rings, Proc. Amer. Math. Soc., 8 (1957), 1093-1100. 
[15] I.S. Rakhimov, K.K. Dey, A.C. Paul, Prime gamma-near-rings with $(\sigma, \tau)$ derivations, Inter. J. of Pure and Applied Mathematics, To Appear.

[16] B. Satyanarayana, A note on $\Gamma$ - Near Rings, Indian J. of Math., 41, No. 3 (1999), 427-433

[17] B. Satyanarayana, A note on $\Gamma$-near rings, Japan Acad. Ser. A Math. Sci., 59, No. 8 (1983), 382-383

[18] M. Uckun, M.A. Ozturk, Y.B. Jun, On prime gamma near-rings with derivations, Commun. Korean Math. Soc., 19, No. 3 (2004), 427-433.

[19] M. Uckun, M. A. Ozturk, On the trace of symmetric bi-gamma derivations in gamma near-rings, Houston Journal, 33, No. 2 (2007), 323-339.

[20] X.K. Wang, Derivations in prime near-rings, Proc. Amer. Math. Soc., 121, No. 2. 
